\section{Asthma und COPD haben Einfluss auf Pathogenese von Rheuma}

Ford JA et al. Asthma, Chronic Obstructive

Pulmonary Disease, and Subsequent Risk for

Incident Rheumatoid Arthritis Among Women:

A Prospective Cohort Study. Arthritis Rheumatol

2020; 72: 704-713

Die Pathogenese der rheumatoiden Arthritis (RA) kann mit Atemwegsmanifestationen einhergehen. Die Rolle chronischer Atemwegserkrankungen bei der RA-Entwicklung ist jedoch unklar. USamerikanische Ärzte haben untersucht, ob Asthma und chronisch obstruktive Lungenerkrankung (COPD) jeweils mit RA assoziiert sind.

Zur Klärung dieser Fragen konzipierten die Autorinnen und Autoren eine prospektive Kohortenstudie mit gepoolten Daten der Nurses' Health Study von 1988-2014 (NHS) und von 1991-2015 (NHS II). Das Alter der Studienteilnehmerinnen von NHS lag zwischen 30 und 55 Jahren, das der Teilnehmerinnen von NHS II zwischen 25 und 42 Jahren. Erfasst wurden selbst gemeldete, ärztlich diagnostizierte Asthma- oder COPD-Erkrankungen, die durch validierte Zusatzfragebögen bestätigt wurden. Primäres Ergebnis war ein RA-Ereignis, das durch Überprüfung der Krankenakte durch 2 Rheumatologen bestätigt wurde. Kovarianten, einschließlich des Raucherstatus, gemessen mit dem Verbrauch von Zigarettenpackungen/Jahr wurden im 2-jährigen Rhythmus überprüft.

Die Gesamtstudienpopulation unter Einbeziehung der Asthma- und der COPD-Analyse ergab eine Stichprobengröße von mehr als 200000 Frauen. Darunter waren mehr als 15000 Frauen mit bestätigter Asthmadiagnose, mehr als 3500 Frauen mit COPD und 1060 Fälle inzidenter rheumatoider Arthritis (63\% seropositiv) und zwar in einem Nachuntersuchungszeitraum von 4384471 Personenjahre.

Die Frauen in der COPD-Gruppe waren insgesamt älter als diejenigen in der Asthmaund der Gruppe ohne Asthma und COPD, und sie waren häufiger postmenopausal. Im Vergleich zu Frauen ohne Asthma und COPD hatten diejenigen mit Asthma ein erhöhtes
RA-Risiko (HR 1,53 [95\% KI 1,24-1,88]). Asthma war dabei sowohl mit seropositiver RA als auch seronegativer RA assoziiert, wobei das Risiko zwischen diesen beiden Gruppen vergleichbar war. Die Stratifizierung nach dem Raucherstatus ergab sowohl für Raucher als auch für Nichtraucher, eine erhöhte Risikoassoziation von Asthma und RA.

COPD war ebenfalls mit einem erhöhten RA-Risiko assoziiert (HR 1,89 [95\% Cl 1,31$2,75]$ ), wobei das Risiko von COPD und seropositiver RA deutlich höher war als das von COPD und seronegativer RA. Ein Zusammenhang von COPD mit rheumatoider Arthritis war dabei in der Untergruppe der Raucher im Alter von > 55 Jahren am deutlichsten ausgeprägt (HR 2,20 [95\% KI 1,383,51]).

FAZIT

Asthma und COPD sind beide mit einem erhöhten Risiko für RA-Ereignisse assoziiert, und zwar unabhängig vom Raucherstatus, der Intensität des Rauchens sowie weiteren potenziellen Kovarianten, fassen die Autorinnen und Autoren ihre Studienergebnisse zusammen. Ihre Ergebnisse stützen die Hypothese, dass chronische Atemwegsentzündungen für die RA-Pathogenese von entscheidender Bedeutung sein könnten.

Richard Kessing, Zeiskam 